Original Research Paper

\title{
Interpretation of Double Langmuir Probe I-V Characteristics at Different Ionospheric Plasma Temperatures
}

\author{
Shankar Bhattarai \\ Department of Physics, Patan Multiple Campus, Tribhuvan University, Lalitpur, Nepal
}

Article history

Received: $24-07-2017$

Revised: 23-10-2017

Accepted: 06-11-2017

Email: oshobhattarai2@gmail.com

\section{Introduction}

Irvin Langmuir was the first who measured the voltampere characteristics by inserting a single conducting wire into plasma and then using it, he determined the electron temperature and plasma density (Laframboise, 1973). This simple device now a days commonly used in plasma measurements, called the single Langmuir probe. The SLPs have some major drawbacks which are difficult to overcome particularly when the reference electrode is absent or when the plasma potential is not well defined (Abe et al., 2006). Moreover, unless the probe area is sufficient small, it may draw large electronic current by disturbing the discharge conditions when operated close to the space potential which shows that the single probe method is not suitable for decaying

\begin{abstract}
Where the plasma potential is fluctuating significantly, Single Langmuir probe method is not relevant to determine the plasma characteristics. This weakness was overcome by the development of the floating double probe. A distortion of the probe characteristics due to varying potential is eliminated, while the whole probe system is floating. This supports probe operation also in discharges with strongly varying potential. Double Langmuir probes provide valuable information on the behavior of space plasmas including ionospheres and the interstellar medium. This research paper focuses on the study of Spherical Double generate the exact plasma conditions of the experimental testing environments computational procedures is adopted. The investigations address the development of a technique to model Maxwellian plasma. Three different ionospheric plasma temperatures are theoretically taken and its effects on floating potential are studied in this research. The variation of floating potential and ion saturation current due to temperature is clearly depicted. A noticeable trail in the I-V curves is the bump that occurs right after the floating potential. This feature in the transition region affects ability to determine the electron temperature, ion saturation current and plasma potential. Symmetric characteristic when both tips are of equal geometry is an important advantage of the double contaminated with deposits, so also does any probe. Here I have also deliver some sense of how one might proceed to use these results in the analysis of experimental I-V curves acquired in space.
\end{abstract}

Keywords: Double Langmuir Probe, I-V Characteristic, Maxwellian Plasma, Ionosphere 
where spatial resolution in the axial and radial directions suffers severely due to probe operating restrictions; i.e. independent electrode sheaths. A symmetric double probe is selected over an asymmetric double probe because the symmetry of the discharge chamber and the simplicity in data analysis outweighs the benefits gained by sampling more of the electron energy distribution (Piel et al., 2001). In analytic treatment of Double Langmuir probe, electron velocities are assumed to follow a Maxwell-Boltzmann distribution. As current flowing to each probe depends on the balance between ions and electrons collection due to individual probe bias, flow of plasma or presence of any energetic component of electrons or ions in plasma may have an effect on interpretation of probe analysis.

The symmetric double probe is sized as small as possible in order to allow spatially resolved measurements similar to the Single Langmuir probe. The use of ceramic, in this case alumina, is necessitated by the high temperatures in the plasma. The electrodes of the double probe are sized similar to the single Langmuir probe electrode (Oyama and Hirao, 1976). Since the measured current is fixed at the ion saturation current. While the double probe electrodes consist of slightly thicker tungsten wire with longer length in order to increase the measured current and accuracy of the measured parameters.

\section{The Conventional Langmuir Probe Technique for Maxwellian Electrons}

Probes are classically classified according to geometry and number of electrodes. A probe may have any geometry, but cylindrical, spherical, and planar probes are usually employed because of their symmetry. The commencement for electron temperature $T_{e}$ measurements is the conventional Langmuir probe theory of Mott-Smith and Langmuir, supposing a Maxwellian energy distribution, $F(E)$, given by (Oyama and Hirao, 1976):

$$
F(E) \propto \sqrt{E} \exp \left(\frac{-E}{k T_{e}}\right)
$$

where, $k$ is the Boltzmann constant. Hence, in a Maxwellian plasma, when the probe to plasma potential, $V$, is driven negative, $I_{e}$ decreases exponentially:

$$
I_{e}=I_{e s} \exp \left(\frac{e V}{k T_{e}}\right)
$$

where, $I_{\mathrm{es}}$ is $A N_{e} e\left(k T_{e} / 2 \pi m_{e}\right)^{1 / 2}$ stands for the random electron current, $e$ is the electron charge, $m_{e}$ is electron mass, and $A$ is the probe area. This equation is suitable for all probe geometries for explaining the electron retarding region in a Maxwellian plasma (Oyama and Hirao, 1976). By appropriate the electron retarding region with an exponential function, the derivation of $T_{e}$ is usually done. While some times $T_{e}$ is obtained electronically by computing the ratio of the first and second derivatives of $I_{e}$ in spite of analysis of the I-V curves.

For a Maxwellian plasma, the electron saturation regions differ greatly with collector geometry. For Planar and Spherical Langmuir probe electron saturation regions extremely depends on $I_{e}$. However for the cylindrical probe electron saturation region is nearly independent. So, electron saturation region is highly temperature dependent for the planar and spherical probes. Due to the large collecting surface area, cylindrical and spherical probes have the advantage of producing huge saturation currents for the same density, an advantage for measurements in regions of very low density (Bernstein and Rabinowitz, 1959).

\section{The Electron Saturation Current for Typical Langmuir Probe}

The Schematic circuit diagram of Double Langmuir Probe is shown in Fig. 1. By biasing the probe with positive voltage, the electrons and negative ions are attracted to the probe and electron saturation occurs. Figure 2 and 3 shows the differences between Double and Single Langmuir probe Current-voltage characteristic graphically. The electron saturation region and is used to determine the electron density of the plasma. All electrons with $V_{x}$ component toward probe are collected in the electron saturation current (Demchenko et al., 1974):

$$
\begin{aligned}
& I_{e s}=-n e A \int_{0}^{\infty} d v_{x} v_{x}\left(\frac{2 \pi K T_{e}}{m_{e}}\right)^{-1 / 2} \exp \left(\frac{-\frac{1}{2} m_{e} v_{x}^{2}}{K T_{e}}\right) \\
& I_{e s}=-n e A\left(\frac{K T_{e}}{2 \pi m_{e}}\right)^{1 / 2}
\end{aligned}
$$

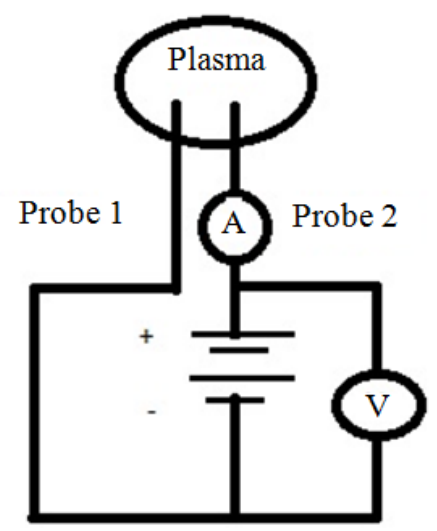

Fig. 1: Typical double Langmuir probe circuit 


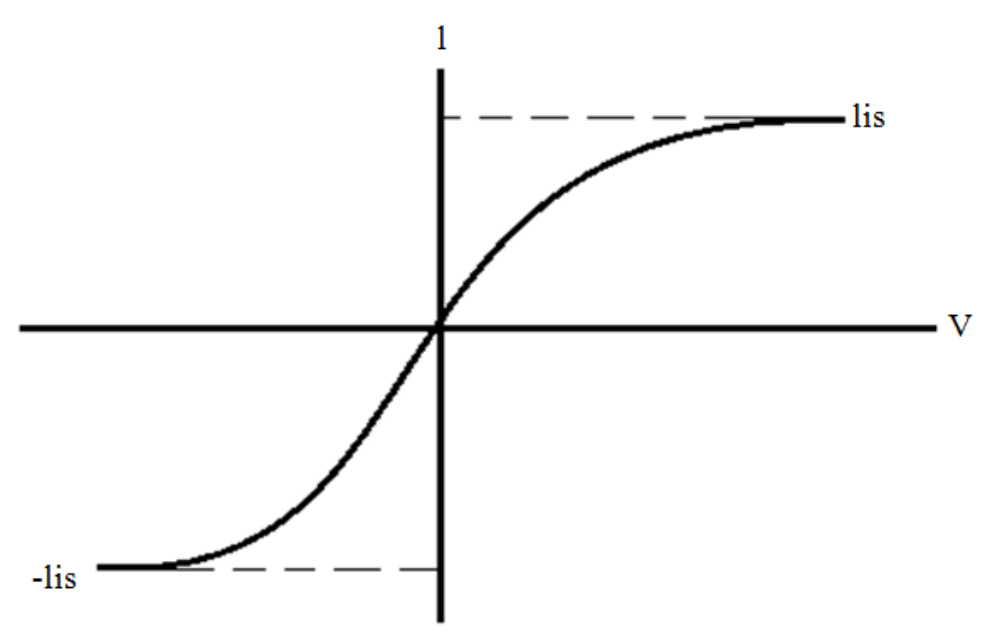

Fig. 2: Current-voltage characteristic of double probe

When electron are resisted, the total current is (Demchenko et al., 1974):

$$
I=I_{i s}-n e A \int_{v_{\min }}^{\infty} d v_{x} v_{x}\left(\frac{2 \pi K T_{e}}{m_{e}}\right)^{-1 / 2} \exp \left(\frac{-\frac{1}{2} m_{e} v_{x}^{2}}{K T_{e}}\right)
$$

Substituting, $\frac{1}{2} m_{e} v_{\min }^{2}=-e V$ :

$$
I=I_{i s}-n e A\left(\frac{K T_{e}}{2 \pi m_{e}}\right)^{1 / 2} \exp \left(\frac{e V}{K T_{e}}\right)
$$

This is the basis equation which clearly shows the relationship between electron current and probe voltage. This demonstrations that the electron current increases exponentially until the probe voltage is the same as the plasma space potential.

\section{The Ion Saturation Current for Typical Langmuir Probe}

Further biasing the probe increasingly with negative voltage, a point is reached where no electron is able to reach to the probe, only positive ions are collected. This section is called the ion saturation region and is used to govern the ion density and ion temperature of the plasma. The ion saturation current is then given as:

$$
I_{i s}=n e A\left(\frac{2 K T_{e}}{m_{i}}\right)^{1 / 2}
$$

where, $A$ is the area of the probe used. It depends on the surface area of the probe, which was implied for the plasma diagnostic.

\section{Floating Potential}

Where no net current flows through the probe and there is a balance between electron and ion current, giving zero net current is called the floating potential (Seiichi, 1971). Consider the floating potential, when $V$ $=V_{f}$, the ion and electron currents are equal and the net probe current is zero:

$V_{f}=-\frac{K T_{e}}{e} \ln \left(\frac{m_{i}}{4 \pi m_{e}}\right)^{1 / 2}$

It is the point on the current-voltage curve where the current drawn by the probe is zero. With the help of floating potential we can easily understood the currentvoltage relationship metaphorically.

\section{The Electron Temperature}

The measurement of the electron temperature can be obtained from Equation (6). For $I_{i s}<I$, we have:

$$
\begin{aligned}
& I=-n e A\left(\frac{K T_{e}}{2 \pi m_{e}}\right)^{1 / 2} \exp \left(\frac{e V}{K T_{e}}\right) \\
& I=I_{e s} \exp \left(\frac{e V}{K T_{e}}\right) \\
& \frac{d I n|I|}{d V}=\frac{e}{K T_{e}}
\end{aligned}
$$

This equations shows that the relationship between electron current and electron saturation current with plasma temperatures and applied voltage (Demchenko et al., 1974). Through differentiating the logarithm of the electron current with respect to the probe voltage $V$; on behalf of $V<0$, the electron temperature is achieved 
(Beal et al., 2012). We memo that, the distribution is a Maxwellian when the slope of $\ln I$ vs. $V$ is a straight line.

\section{Theory of Double Langmuir Probe}

The double Langmuir probe alignment, with two electrodes, floats with the plasma and electrodes are swept relative to the floating potential. Double probe system eradicates the need for a fixed reference potential, limits biased electrode collected current to the ion saturation current, diminishes local plasma perturbations with a net zero current to the electrode pair, and lessens the electrode voltage arch (Smith and Overzet, 1998). This has several advantages over the single probe geometry. The symmetric double probe is sized as small as possible in order to allow spatially resolved measurements similar to the single Langmuir probe. The use of ceramic, in this case alumina, is necessitated by the high temperatures in the plasma. The electrodes of the double probe are sized similar to the single Langmuir probe electrode. Because the measured current is fixed at the ion saturation current, the double probe electrodes consist of slightly thicker tungsten wire with longer length in order to increase the measured current and accuracy of the double probe plasma parameters measured (Yong-Ik et al., 2002).The probe 1 current collection is express as:

$$
I_{1}=I_{1 i s}-I_{1 e s} \exp \left(\frac{e\left(V_{1}+V_{f}-V_{s}\right.}{K T_{e}}\right)
$$

From the description of floating potential (Demchenko et al., 1973):

$$
\begin{aligned}
& I_{e s} \exp \left(\frac{e\left(V_{f}-V_{s}\right)}{K T_{e}}\right)=I_{i s} \\
& I_{1}=I_{1 i s}\left[1-\exp \left(\frac{e V_{1}}{K T_{e}}\right)\right] \\
& I_{2}=I_{2 i s}\left[1-\exp \left(\frac{e V_{2}}{K T_{e}}\right)\right]
\end{aligned}
$$

When the probe area are equal then, $I_{1 i s}=I_{2 i s}=I_{i s}$. From the explanation of zero net probe current, $I=I_{1}=-I_{2}$. Combining this all:

$$
\frac{I-I_{i s}}{-I-I_{i s}}=\exp \left(\frac{e\left(V_{1}-V_{2}\right)}{K T_{e}}\right)
$$

For, the double Langmuir probe, potential is defined by $\phi=V_{1}-V_{2}$. Then we get:

$$
I=-I_{i s} \tanh \left(\frac{e \varphi}{2 K T_{e}}\right)
$$

After differentiating with respect to potential $\phi$ :

$\frac{d I}{d \varphi}=-I_{i s}\left(\frac{e}{2 K T_{e}}\right)$

Latest theoretical studies of double Langmuir probe characteristics in low-temperature plasma commanded to development of a new analysis method that employs analytical fits to Laframboise's numerical results and circuit analysis to verbalize relationships between applied electrode voltage, current collection, and the plasma properties (Menart and Shang, 2003).

\section{Results and Discussion}

Assuming electron velocity distribution is not deviating substantially from a Maxwellian, theoretical analysis and interpretation of the Double Langmuir Probe (DLP) for bulk electron temperature measurement is presented. This exertion is typically based on the I-V characteristic of DLP. The main propose of this effort is to study the Current-Voltage characteristic of spherical Langmuir probe and to analyzed the effect of temperature on width of electron retardation regions. The methodology of our study includes the theoretical derivation of the relationships between various parameters and then plotting the parameters in appropriate range from a mathematical software. The study predicts conventional expression from standard DLP theory to give overrated value of electron temperature.

\section{The Variation of Double Langmuir Probe I-V Curves for Different Ionospheric Temperatures}

This research is typically based on lower ionosphere. The $I-V$ characteristic is a essential part of diagnosis of plasma parameters So, it is illustrated in the figure. For this purpose, it is assumed that density, $N_{e}=10^{16} \mathrm{~m}^{-3}, r_{p}$ $=10 \mathrm{~mm}$ and the ionospheric temperature are 1000, 2000,3000 and $4000 \mathrm{~K}$. These figures demonstrate the effect of temperature on the width of the electron retardation regions.

The above computational analysis, Fig. 4 shows that, when the plasma temperature is $1000 \mathrm{~K}$, the ion saturation current is -0.022 microampere in the probe, and floating potential is nearly -0.4 V. Figure 5 demonstrations, when the DLP is immersed in the $2000 \mathrm{~K}$ ionospheric plasma, at the same density and other conditions the ion saturation current is -0.031 microampere, floating potential is $-0.7 \mathrm{~V}$. It can be accomplish that at higher plasma temperature the ion saturation current and floating potential in the probe is inferior as comparative to lower ionospheric plasma temperature. Figure 6 and 7 reports similar results to the previous case. 


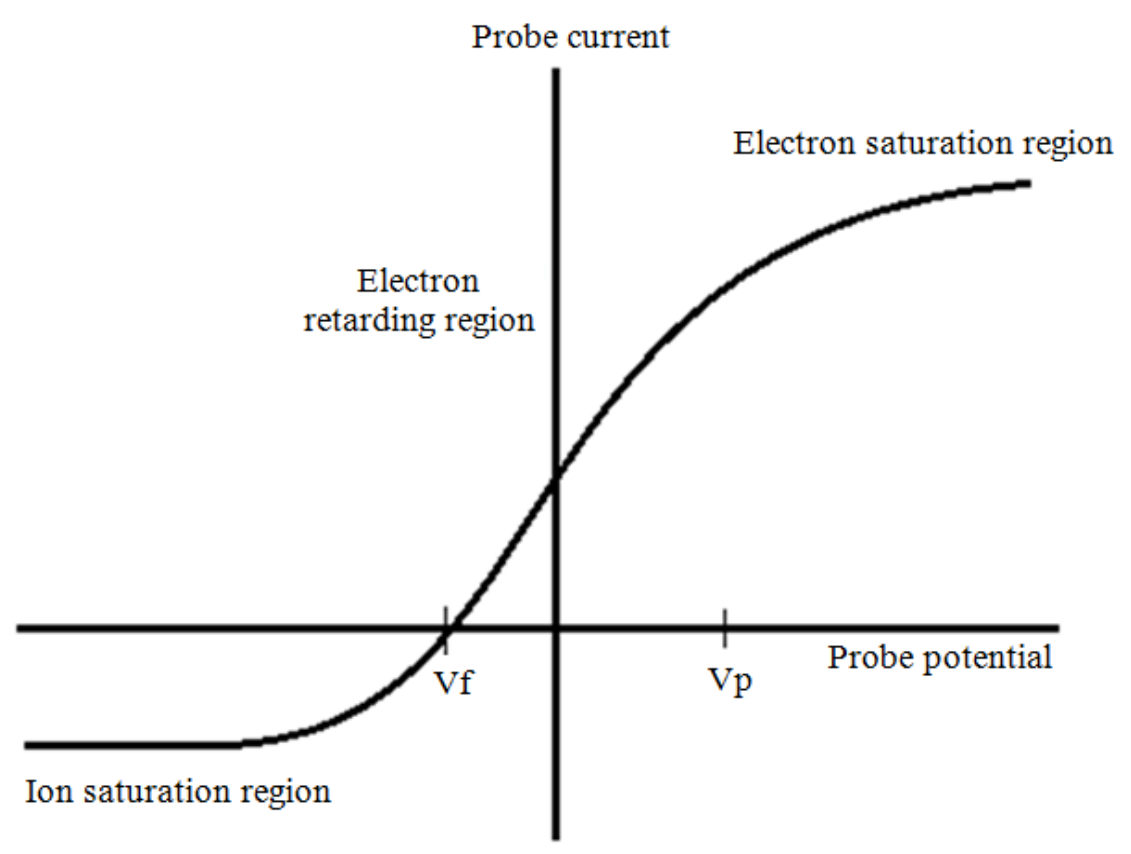

Fig. 3: A typical current-voltage curve for a single Langmuir probe

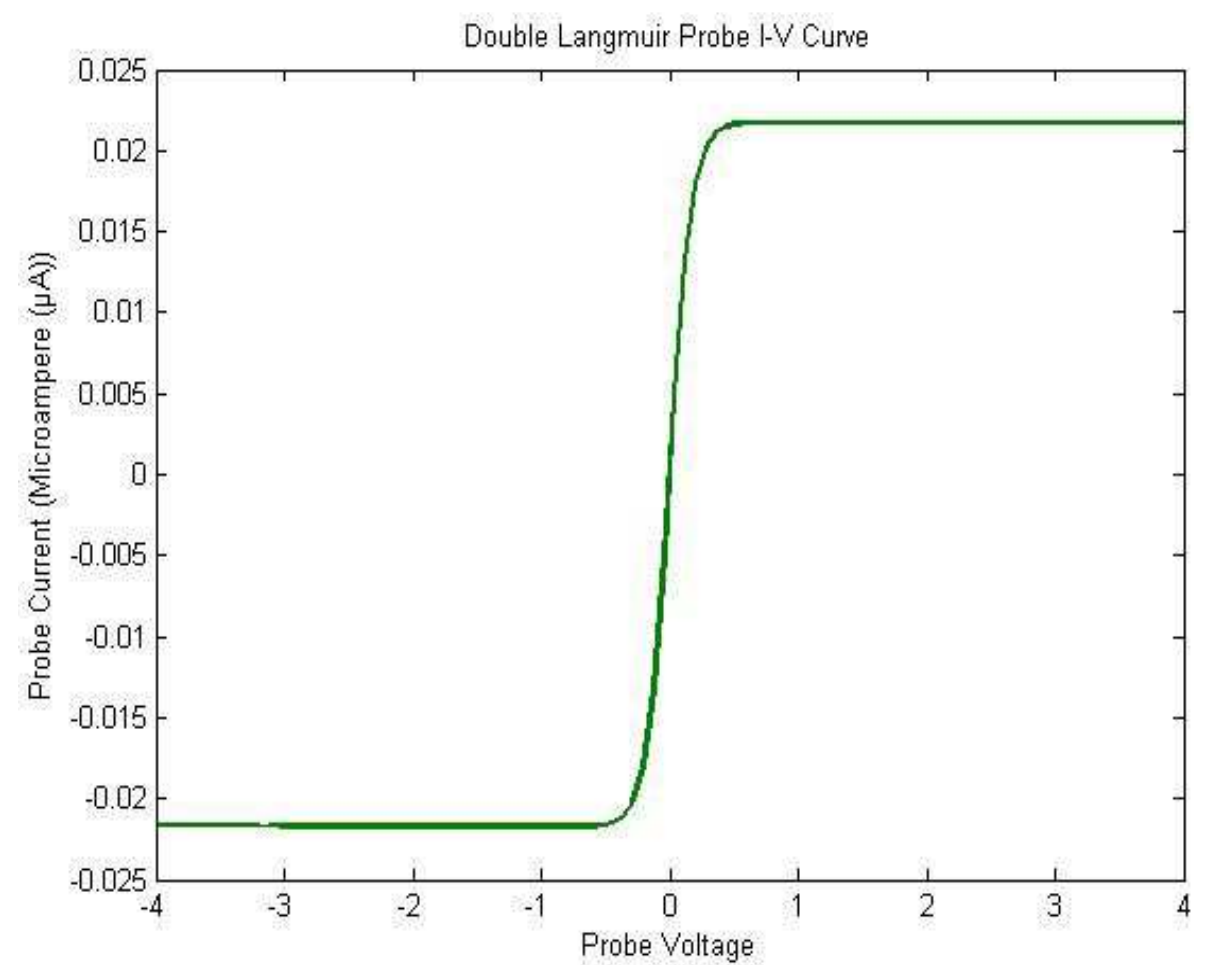

Fig. 4: Double langmuir probe I-V curve at 1000K

To sum up, it is perceived that although the graphical representation of the results obtained by the Double Langmuir probe and SLP methods shows the similar variation with trend but the electron temperature obtained by the double Langmuir probe method is comparatively higher while the electron number density is relative lower than that obtained by the Single Langmuir probe technique. 


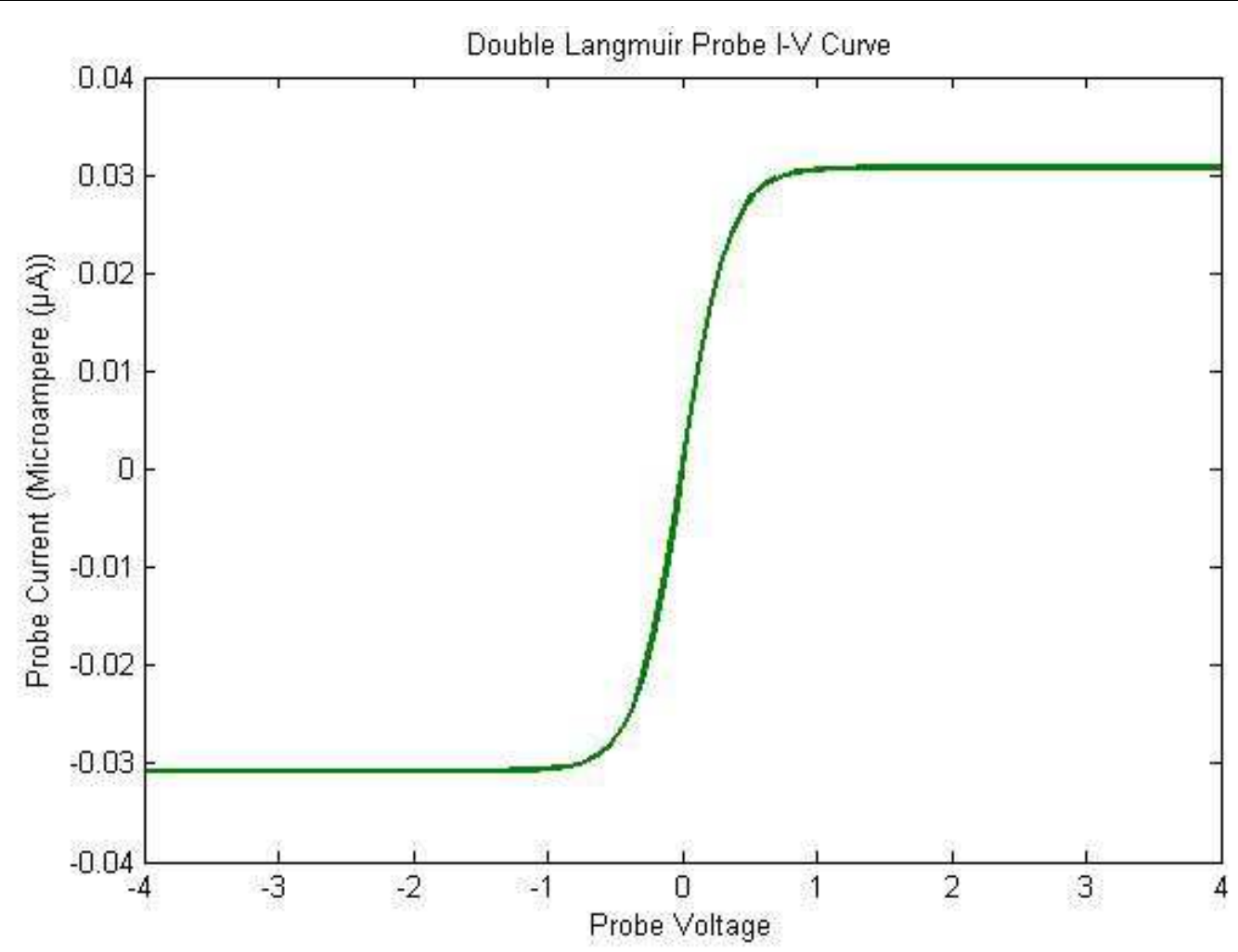

Fig. 5: Double Langmuir probe I-V curve at $2000 \mathrm{~K}$

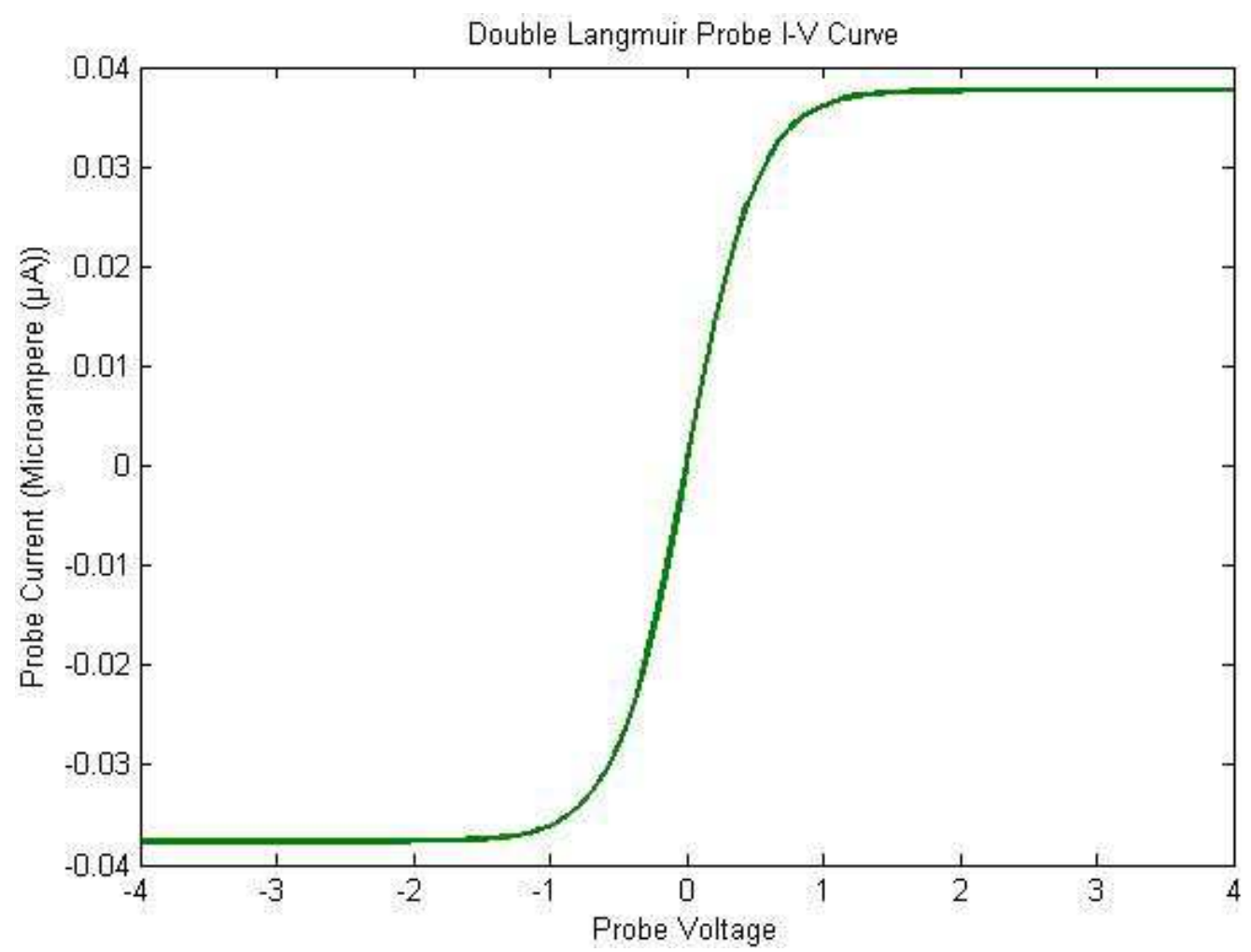

Fig. 6: Double Langmuir probe I-V curve at 3000K 


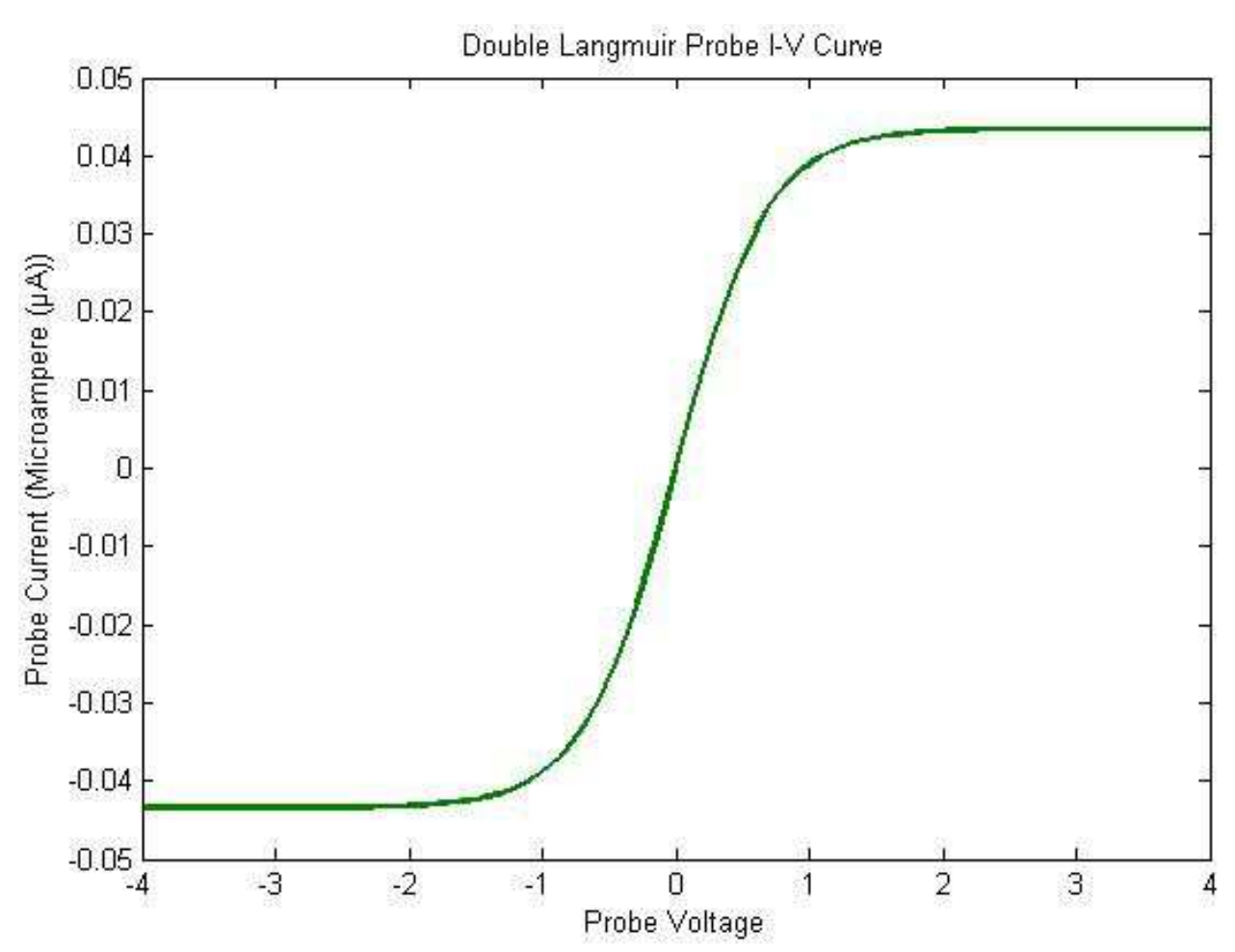

Fig. 7: Double Langmuir probe I-V curve at 4000K

\section{Conclusion}

The Double Langmuir Probe provides an instantaneous measurement diagnostic of space plasma parameters. Langmuir probes, in principle, provide a simple and relatively inexpensive diagnostic for measuring the plasma parameters and its characteristic. The $I-V$ characteristic of the DLP in the Maxwellian Space plasma at $1000 \mathrm{~K}$ to $4000 \mathrm{~K}$ temperature and its effect on the variation of floating potential, which are intrigued in graph by using the computational software by putting the seemly ranges for various parameters. All elementary processes were extensively considered and most of information has been presented. The main objective of this research work was to improve a Langmuir probe instrument for sounding rockets capable of accomplishment high-speed absolute electron density measurements, and in that way be able to detect ionospheric plasma density structures; when the potential is fluctuating significantly. Though this work has presented and illustrated the Double Langmuir Probe theory for Maxwellian electron populations. The Double Langmuir Probe can collect a maximum current equal to the ion saturation current and does not divert the plasma as much as the Single Langmuir probe.

\section{Acknowledgement}

I am very much grateful to my Master Degree Dissertation Supervisor Assoc. Prof. Dr. Lekha Nath
Mishra, Head, Department of Physics, Patan Multiple Campus Tribhuvan University, Patandhoka Lalitpur Nepal and my father Assoc. Prof. Dr. Yuga Raj Bhattarai, BBA Director, Department of Management, Patan Multiple Campus, Tribhuvan University, Patandhoka Lalitpur Nepal, for the encouragement and their support throughout the research.

\section{Ethics}

Authors declare that are not ethical issues that may arise after the publication of this manuscript. This article is original and contains unpublished material.

\section{References}

Laframboise, J.G., 1973. Probe design for orbit limited current collection. Phys. Fluids, 16: 629-629.

Abe, T., K. I. Oyama and A. Kadohata, 2006. Electron temperature variation associated with the auroral energy input during the DELTA campaign. Earth Planets Space, 58: 1139-1146.

Peterson, E.W. and L. Talbot, 1970. Collisionless electrostatic single-probe and double-probe measurements. AIAA J., 8: 2215-2219.

Piel, A., M. Hirt and C.T. Steigies, 2001. Plasma diagnostics with Langmuir probes in the equatorial ionosphere: I. The influence of surface contamination. J. Phys. D: Applied Phys., 34: 2643-2649. 
Oyama, K. and K. Hirao, 1976. Application of a glasssealed Langmuir probe to ionosphere study. Rev. Sci. Instrum, 47: 101-107.

Bernstein, I.B. and I.N. Rabinowitz, 1959. Theory of electrostatic probes in a low-density plasma. AIP Phys. Fluids, 2: 112-121.

Seiichi, A., 1971. Effect of electron and ion drifts on double probe. J. Phys. Society Japan, 31: 1221-1231.

Beal, B.E., L. Johnson, D.L. Brown, J.M. Blakely and D. Bromaghim, 2012. Improved analysis techniques for cylindrical and spherical double probes. Rev. Sci. Instrum.

Smith, B.A. and L.J. Overzet, 1998. Improvements to the floating double probe for time-resolved measurements in pulsed RF plasmas. Rev. Scientific Instruments, 69: 1372-1372.
Yong-Ik, S., H.B. Lim and R.S. Houk, 2002. Diagnostic studies of low-pressure inductively coupled plasma in argon using a double Langmuir probe. J. Anal. At. Spectrom. 17: 565-569.

Demchenko, P.A., L.I. Krupnik and N.G. Shulika, 1973. Analysis of voltage-current characteristics of an electric double probe. Soviet Phys. Technical Phys., 18: 1054-1056.

Menart, J. and J. Shang, 2003. Data reduction analysis for cylindrical, double Langmuir probes operating in collision less to Collisional, quiescent plasmas. Proceedings of the 41st Aerospace Sciences Meeting and Exhibit, Jan. 6-9, Reno, Nevada. pp: 1-1.

DOI: $10.2514 / 6.2003-136$ 